\title{
Epidemiologia da leishmaniose canina no município de Pedro II, Piauí, entre os anos de 2013 e 2019
}

\author{
Lucimary do Nascimento \& Etielle Barroso de Andrade (i)
}

Grupo de Pesquisa em Biodiversidade e Biotecnologia do Centro-Norte Piauiense - BIOTECPI, Instituto Federal de Educação, Ciência e Tecnologia do Piauí - IFPI - Campus Pedro II, Rua Antonino Martins de Andrade 750, Engenho Novo, 64255000, Pedro II, Piauí, Brasil. E-mail: etlandrade@ hotmail.com

Nascimento L. \& Andrade E.B. (2021) Epidemiologia da leishmaniose canina no município de Pedro II, Piauí, entre os anos de 2013 e 2019. Pesquisa e Ensino em Ciências Exatas e da Natureza, 5: e1623. http://dx.doi.org/10.29215/pecen.v5i0.1623

Editor acadêmico: Samuel Vieira Brito. Recebido: 27 outubro 2020. Aceito: 13 janeiro 2021. Publicado: 19 janeiro 2021

Resumo: A leishmaniose é um grave problema de saúde pública e os cães são considerados como a principal fonte de infecção em áreas urbanas. O município de Pedro II possui grande número de casos de leishmaniose humana, mas não há divulgação sobre a doença em cães. Aqui, determinamos a ocorrência de leishmaniose canina nas zonas urbana e rural do município de Pedro II, estado do Piauí, entre 2013 e 2019. Os dados foram fornecidos pela Coordenação de Controle de Endemias do município. Foram registrados 899 casos caninos da doença, dos quais $51 \%$ ocorreram na zona urbana, principalmente nos bairros próximos às áreas de vegetação. Do total, 287 cães foram eutanasiados representando uma taxa de eliminação de 32\%. Os anos de 2018 e 2019 apresentaram os maiores números de casos. Apesar de não haver correlação entre o número de cães infectados e os casos em humanos, Pedro II possui um elevado número de cães infectados, além de áreas favoráveis ao desenvolvimento do vetor. Assim, apresentamos informações básicas sobre a leishmaniose canina no município que podem ser usadas para direcionar medidas de controle e proteção dos animais e humanos, além de contribuir com informações da leishmaniose na região norte do estado.

Palavras chave: Zoonose, epidemiologia, leishmaniose visceral, flebotomíneos, saúde pública.

Epidemiology of canine leishmaniasis in the municipality of Pedro II, Piauí, between the years 2013 and 2019

Abstract: Leishmaniasis is a serious public health problem and dogs are the main source of infection in urban areas. The municipality of Pedro II has a large number of human leishmaniasis cases, but there is no divulgation about the disease in dogs. Herein, we determined the occurrence of canine leishmaniasis in the urban and rural areas of the Pedro II municipality, state of Piauí, between 2013 and 2019. The data were provided by the municipality's Endemic Control Coordination. We recorded 899 cases of canine leishmaniasis, of which $51 \%$ occurred in the urban area, mainly in neighbourhoods close to vegetation areas. Of the total, $287 \mathrm{dogs}$ were euthanized representing a $32 \%$ elimination rate. The years 2018 and 2019 had the highest number of cases. Although there is no correlation between canine and human leishmaniasis, the Pedro II municipality has a high number of infected dogs, in addition to areas favourable to the vector development. Thus, we present basic information about canine leishmaniasis in the Pedro II municipality that can be used to directly control and protection measures for animals and humans, in addition to contributing with information on leishmaniasis in the northern region of the state.

Key words: Zoonosis, epidemiology, visceral leishmaniasis, sandflies, public health.

\section{Introdução}

A Leishmaniose visceral (LV), conhecida também como calazar, é uma doença infecciosa não contagiosa, causada por protozoários flagelados do gênero Leishmania e 
transmitida por meio da picada das fêmeas de flebotomíneos da espécie Lutzomyia longipalpis (Marcondes \& Rossi 2013). Essa doença é considerada uma das sete endemias mundiais de prioridade da Organização Mundial da Saúde (OMS), sendo encontrada em várias regiões do mundo e apontada como um grave problema de saúde pública. É considerada endêmica em mais de 60 países, sendo Índia, Bangladesh, Nepal, Sudão e Brasil os responsáveis por cerca de 90\% dos casos mundiais (Caldas et al. 2013). No Brasil, essa doença atinge cerca de 21 estados, concentrados principalmente na região Nordeste (Siqueira 2012). O clima, temperatura e vegetação contribuem para o aumento no número de casos na região, sendo Bahia, Maranhão, Ceará e Piauí os estados mais afetados pela enfermidade (Bavia et al. 2005).

Além de afetar os humanos, a LV acomete mamíferos domésticos e alguns animais silvestres. A principal fonte de infecção na área urbana são os cães, onde se manifestam na forma visceral, e nas áreas rurais as raposas e gambás (Silva et al. 2005). Os animais apresentam sintomas basicamente parecidos com os seres humanos, e desenvolvem a doença na forma visceral e cutânea. Na visceral os principais órgãos afetados são o fígado, baço, linfonodos e rins, e na forma cutânea o animal apresenta pelagem seca, perda de pelos, unhas grandes e quebradiças (Tilley \& Smith Jr. 2008). Muitos cães não apresentam sintomas, já outros entram em um estado terminal, o que dificulta o cálculo do número de casos (Gatti et al. 2018). Como as manifestações da doença são semelhantes de outras enfermidades é necessário a realização de testes laboratoriais e epidemiológicos para que seja feito um diagnóstico mais complexo que confirme a presença do parasita no animal (Freitas 2011).

No Brasil, a leishmaniose é uma das doenças que mais afeta os cães, muitas vezes não causa sintomas, o que gera maior perigo, pois estes são a principal fonte de transmissão domiciliar da doença para humanos e outros animais. É comum existir infecção em áreas rurais e urbanas, no entanto, nas áreas urbanas verifica-se grande disseminação da doença devido à expansão populacional das cidades, o que leva a sua rápida proliferação deixando a população suscetível à infecção (Gontijo \& Melo 2004). O avanço da área urbana em direção à zona rural fez com que os mosquitos se adaptassem rapidamente ao meio urbano, onde são encontrados próximos a residências que possuem galinheiros, canis e chiqueiros (Brasil 2006).

O Piauí foi o primeiro estado a apresentar surto de leishmaniose em meio urbano no Brasil na década de 80. Esse surto ocorreu devido aos grandes períodos de seca no estado, o que levou a movimentação de pessoas com animais domésticos infectados para locais que não haviam focos da doença (Werneck et al. 2008). No entanto, essa doença é existente no Piauí desde $o$ ano de 1971, no qual era mais predominante na zona rural e rapidamente espalhou-se na zona urbana devido o cão ser o principal reservatório doméstico (Bonates 2003).

O município de Pedro II possui grande número de casos de leishmaniose humana na forma tegumentar e visceral (Batista et al. 2014) e o principal elo de transmissão é por meio de animais domésticos. Considerando o grande número de casos de leishmaniose no município, e a falta de informações relacionadas a doença no município de Pedro II, há a necessidade de pesquisas que evidenciem os casos atuais de leishmaniose canina de modo a permitir maior controle por parte dos órgãos municipais, maior conhecimento por parte da população local, além de medidas para a redução de incidência da doença. Assim, o presente estudo teve como objetivo determinar a ocorrência da leishmaniose canina no município de Pedro II, região Centro-norte do Piauí, entre os anos de 2013 e 2019, comparando a distribuição dos casos entre as zonas urbanas e rurais.

\section{Material e Métodos}

O presente estudo trata-se de uma pesquisa descritiva, quantitativa utilizando dados de leishmaniose canina ocorridos no município de Pedro II, estado do Piauí, entre os anos de 2013 e 2019. O município está localizado na região Centro-Norte do estado do Piauí (04²5'23" S, $41^{\circ} 27^{\prime} 34^{\prime \prime} \mathrm{O}$ ), distante cerca de $200 \mathrm{~km}$ da capital Teresina, possuindo uma área territorial de $1.544 .565 \mathrm{~km}^{2}$ e uma população estimada de 38.778 habitantes para o ano de 2020 (IBGE 2021). Situado na Serra dos Matões, cerca de $600 \mathrm{~m}$ acima do nível do mar e dentro dos limites da Área 
de Preservação Ambiental da Serra da Ibiapaba (Isa 2017; IBGE 2019), o município de Pedro II possui clima serrano e ameno comparado as demais cidades do estado. A região é semiárida, apresentando uma vegetação típica de transição entre a Caatinga e o Cerrado (IBGE 2019). Apresenta clima tropical chuvoso alternadamente úmido e seco, com pluviosidade média anual de $1.000 \mathrm{~mm}$, concentrado entre os meses de dezembro a maio (Aguiar \& Gomes 2004), e temperaturas anuais variando de $18^{\circ} \mathrm{C}$, no período chuvoso, a $30^{\circ} \mathrm{C}$, nos meses de estiagem (Freitas et al. 2016).

Os dados foram coletados junto à Coordenação de Controle de Endemias ligada a Secretaria Municipal de Saúde do município de Pedro II de modo a se obter um panorama sobre a incidência dos casos de leishmaniose canina no município nos últimos anos (2013 a 2019). O período de coleta foi estabelecido de acordo com a disponibilidade dos dados, uma vez que dados anteriores a 2013 não estavam disponíveis. Além dos dados sobre a leishmaniose canina, foi coletado junto à Secretaria Municipal de Saúde informações sobre a leishmaniose tegumentar e leishmaniose visceral em humanos no mesmo período de tempo. Por se tratar de uma pesquisa descritiva com uso de dados disponibilizados pelo setor de epidemiologia do município, não houve a necessidade de aprovação pelo Comitê de Ética no Uso de Animais (CEUA).

Todas as informações descritas nas fichas foram organizadas e tabuladas de modo a obterse o número total de casos confirmados e o número de cães eutanasiados entre os diferentes anos na zona urbana e rural. Após a tabulação dos dados, foi realizada uma comparação do número de casos confirmados, utilizando mapas de distribuição da doença nos diferentes bairros do município. Além disso, foram observados e classificados os bairros com maior número de cães infectados, os períodos de maior ocorrência durante o ano, comparando-os entre zona urbana e rural. A ocorrência dos casos entre os anos analisados e o índice de prevalência foram expressos em forma de gráficos para uma melhor visualização da proporção de casos existentes e de cães acometidos com a doença ao longo dos anos no município. O índice de prevalência foi calculado utilizando o somatório dos casos confirmados do teste rápido e Elisa dividido pelo número total de casos pesquisados.

Um mapa de densidade de Kernel foi produzido para demonstrar as áreas com maior risco de contaminação da doença. Esse método gera uma superfície de densidade para a identificação das áreas com maior número de casos (Lima et al. 2005). Uma vez que não foram disponibilizados dados de localização precisa dos casos na área urbana, geramos uma distribuição aleatória e não sobreposta dos dados usando os números de casos em cada bairro de Pedro II. Os mapas de distribuição dos casos e de densidade de Kernel foram gerados no programa QGIS versão 3.10.6 (QGIS Development Team 2020). Para avaliar a relação entre o número de cães infectados e o número de casos de leishmaniose em humanos, aplicamos um teste de correlação linear de Pearson ( $r$, Zar 2010), com nível de significância de 5\%, usando o programa Past v. 4.03 (Hammer et al. 2001). Para análise de correlação usamos os dados de 2013 a 2018, uma vez que foram disponibilizados dados sobre a leishmaniose humana somente até o ano de 2018.

\section{Resultados}

Foram registrados um total de 899 casos de leishmaniose canina (LC) no município de Pedro II - PI durante todo o período amostrado (2013-2019). Os dados positivos da doença foram encontrados através da soma do teste rápido e do teste laboratorial (teste de ELISA). Houve pouca diferença entre o número de casos brutos de LC da zona urbana e zona rural. Das 46 localidades rurais do município, 36 tiveram registros de LC (Figura 1), sendo notificados 443 casos positivos, representando cerca de $49 \%$ do número total de casos registrados no município. Os maiores números foram registrados em nove localidades rurais (Cipó de Cima, Carnaúbas, Mangabeira, Lapa, Pequis, Lagoa do Sucuruju, Aroeira, Açude dos Mourão e Serra dos Matões) no qual somaram cerca de $50 \%$ dos casos registrados na zona rural (Figura 1). Na zona urbana foram registrados 456 casos de leishmaniose canina, distribuídos em sete bairros correspondendo a aproximadamente $51 \%$ do número total, os maiores números foram 
registrados em apenas três bairros (Santa Fé, Centro e Vila Kolping), no qual somaram juntos cerca de $61 \%$ do número total de casos registrados na zona urbana (Figura 2).

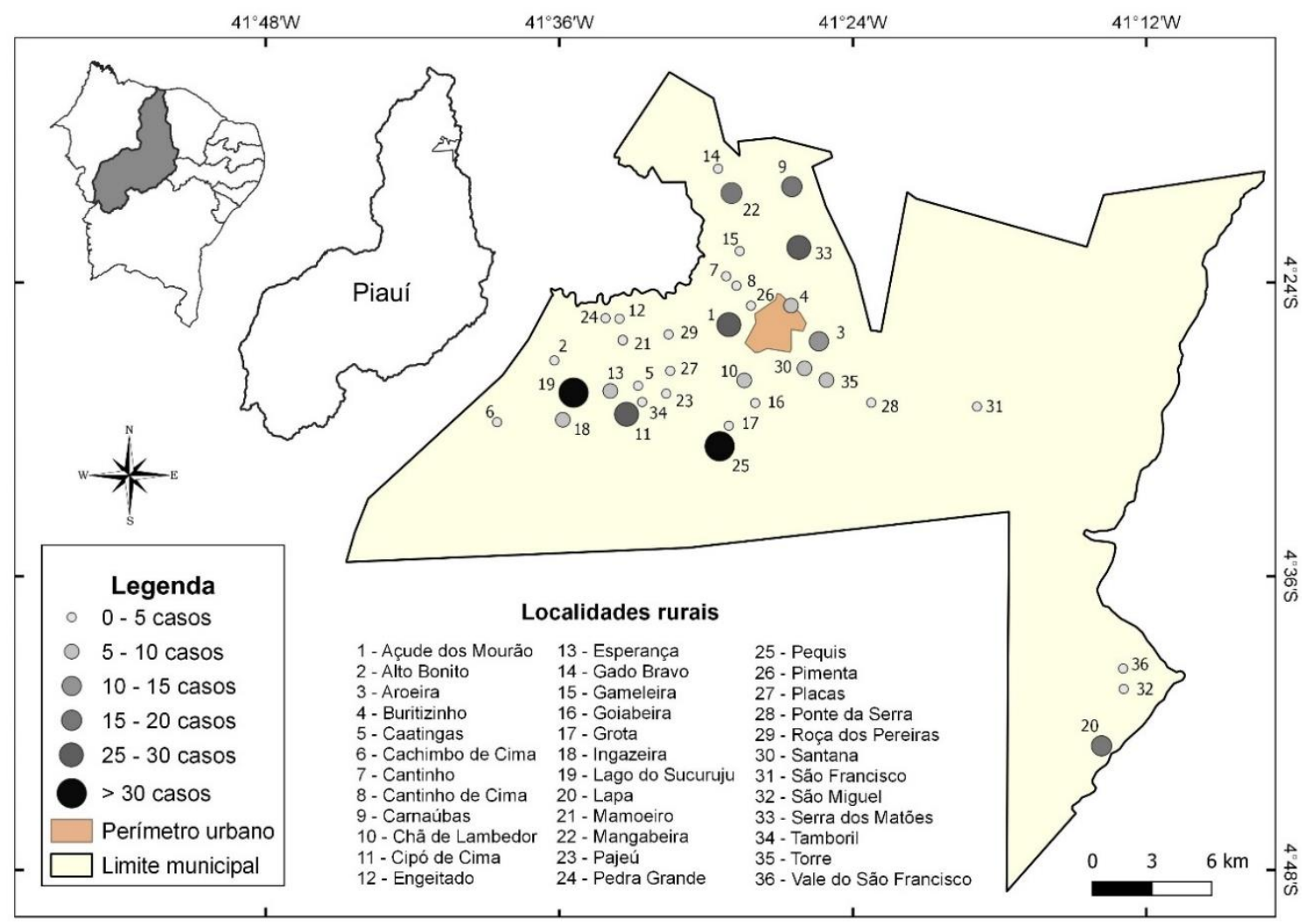

Figura 1. Localização e distribuição de casos de leishmaniose canina nas localidades rurais do município de Pedro II, região norte do estado do Piauí, registrados entre os anos de 2013 a 2019.

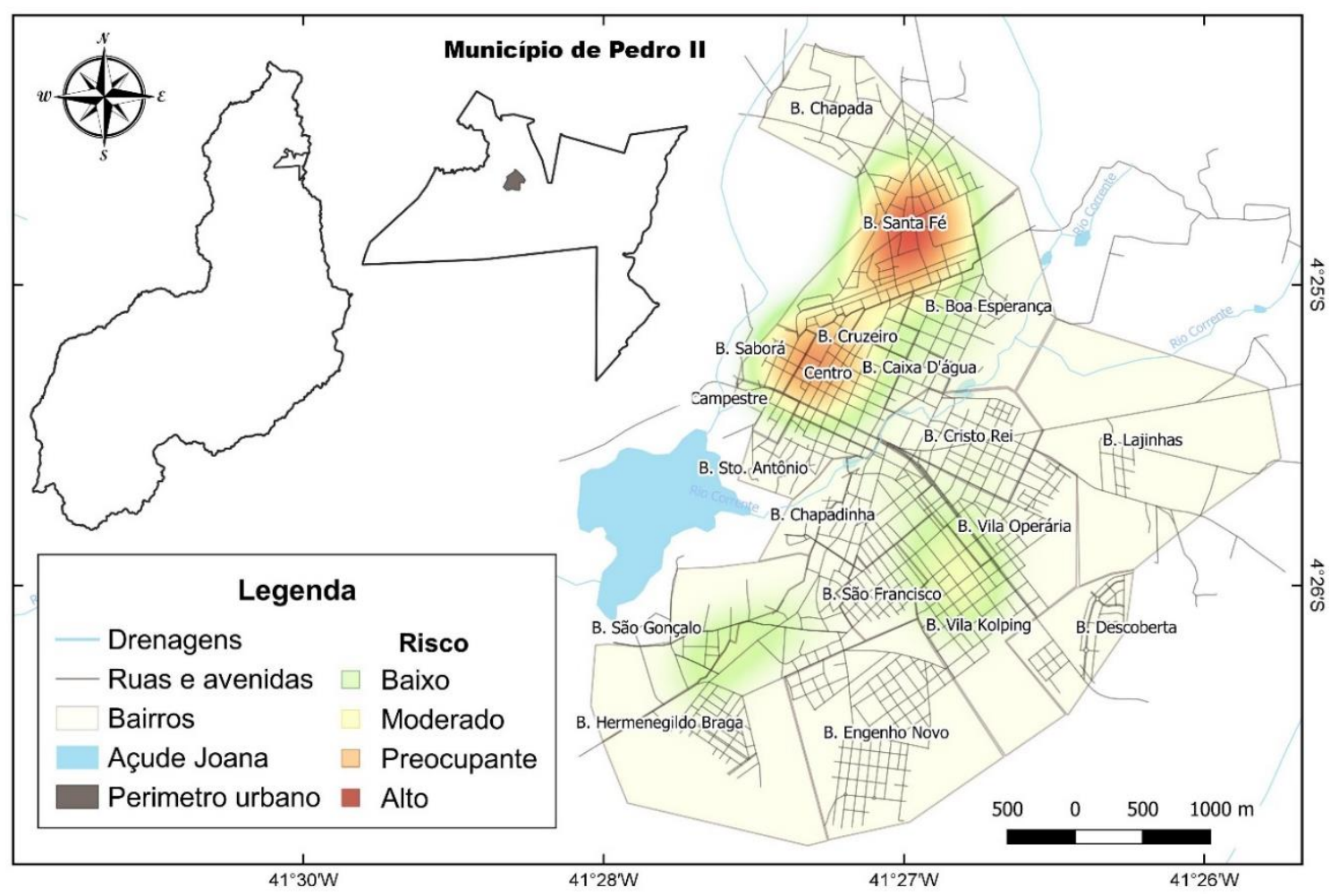

Figura 2. Mapa de risco e distribuição de casos de leishmaniose canina na zona urbana de Pedro II, região norte do estado do Piauí. 
Do total de cães notificados, 287 foram eutanasiados, representando taxa de eliminação de $32 \%$ no período amostrado. Desses, 144 eram da zona rural (16\% dos registros), enquanto que 143 eram da zona urbana (15\% dos registros). Em relação ao período de ocorrência, os anos de 2018 e 2019 apresentaram os maiores números de casos (356 e 150 respectivamente; Tabela 1), sendo 2018 com a maior proporção de casos na zona rural e na zona urbana (201 e 155 respectivamente; Figura 3). De acordo com a Coordenação de Endemias, no ano de 2015 não houve casos da doença na zona urbana e em 2017 não houve a contabilização de casos na zona rural. Levando em conta apenas os dados contabilizados, o ano de 2016 apresentou o menor número de casos para a zona rural, enquanto 2015 foi o menor para a zona urbana (Tabela 1).

Tabela 1. Casos de leishmaniose canina no município de Pedro II, região norte do estado do Piauí, registrados entre os anos de 2013 a 2019, mostrando o número de cães infectados e eutanasiados nas zonas urbana e rural.

\begin{tabular}{cccccc}
\hline Ano & Zona urbana (U) & Cães eutanasiados & Zona rural (R) & Cães eutanasiados & Taxa de eliminação \\
\hline 2013 & 30 & 18 & 78 & 62 & $74.1 \%$ \\
2014 & 14 & 6 & 45 & 13 & $32.2 \%$ \\
2015 & 0 & 0 & 53 & 14 & $26.4 \%$ \\
2016 & 7 & 3 & 30 & 11 & $37.8 \%$ \\
2017 & 136 & 41 & - & - & $30.1 \%$ \\
2018 & 155 & 43 & 201 & 35 & $21.9 \%$ \\
2019 & 114 & 32 & 36 & 9 & $27.3 \%$ \\
Total & 456 & 143 & 443 & 144 & - \\
\hline
\end{tabular}

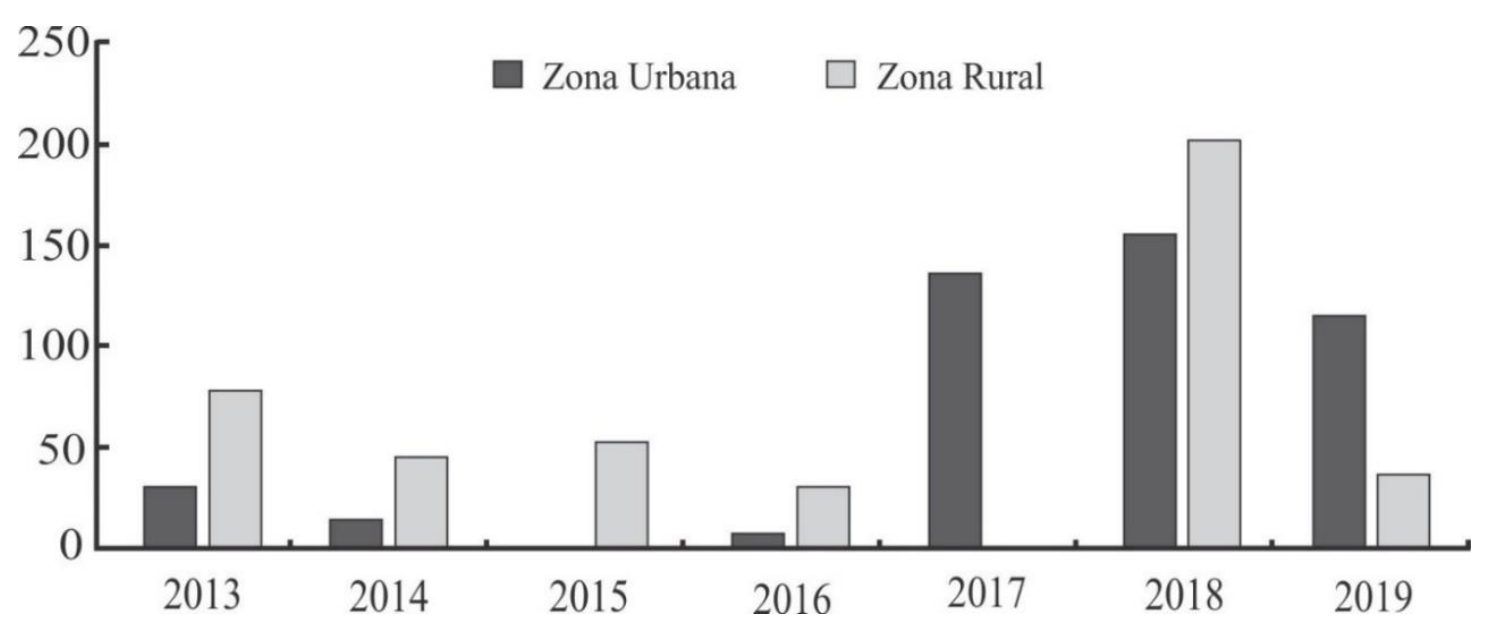

Figura 3. Índice de Ocorrência da leishmaniose canina no município de Pedro II, região norte do estado do Piauí, registrados no período de 2013 a 2019.

Os bairros Santa Fé (141 casos) e Centro (96 casos) apresentaram os maiores números de casos, como mostra a Tabela 2. No entanto, devido ao seu pequeno tamanho, os bairros Saborá, Campestre e Cruzeiro foram contabilizados juntamente com o bairro Centro, assim, como ocorreu com os bairros Chapadinha, Vila Operária e São Francisco, que foram todos contabilizados sob a nomenclatura de Mutirão. Não foram disponibilizados dados detalhados dos bairros nos anos de 2015 e 2016 nem dados completos de todas as localidades nos anos de 2014, 2017 e 2019, assim como os registros sobre raça, sexo, idade, sinais clínicos e procedência do animal. 
Tabela 2. Relação dos bairros e números de casos de leishmaniose na zona urbana do município de Pedro II, região norte do estado do Piauí, registrados entre os anos de 2013 e 2019.

\begin{tabular}{cc}
\hline Bairros & $\mathbf{N}^{\circ}$ de Casos \\
\hline Santa Fé & 141 \\
Centro & 96 \\
Vila Kolping & 42 \\
Areia Branca & 36 \\
Cristo Rei & 6 \\
Mutirão & 15 \\
Boa Esperança & 21 \\
\hline
\end{tabular}

Foi observado maior prevalência de casos registrados no ano de 2018 com $32 \%$ e $26 \%$ na zona urbana e rural, respectivamente, e 2019 com $28 \%$ e $30 \%$ na zona urbana e rural, respectivamente (Figura 4). De acordo com dados disponibilizados pela Secretaria Municipal de Saúde foram registrados 35 casos de Leishmaniose Tegumentar americana (LTA) e 13 de Leishmaniose Visceral entre os anos de 2013 e 2018 (Tabela 3). Apesar do grande número de cães infectados, não houve correlação entre os casos de leishmaniose canina com os casos de leishmaniose em humanos $(p>0.05)$.

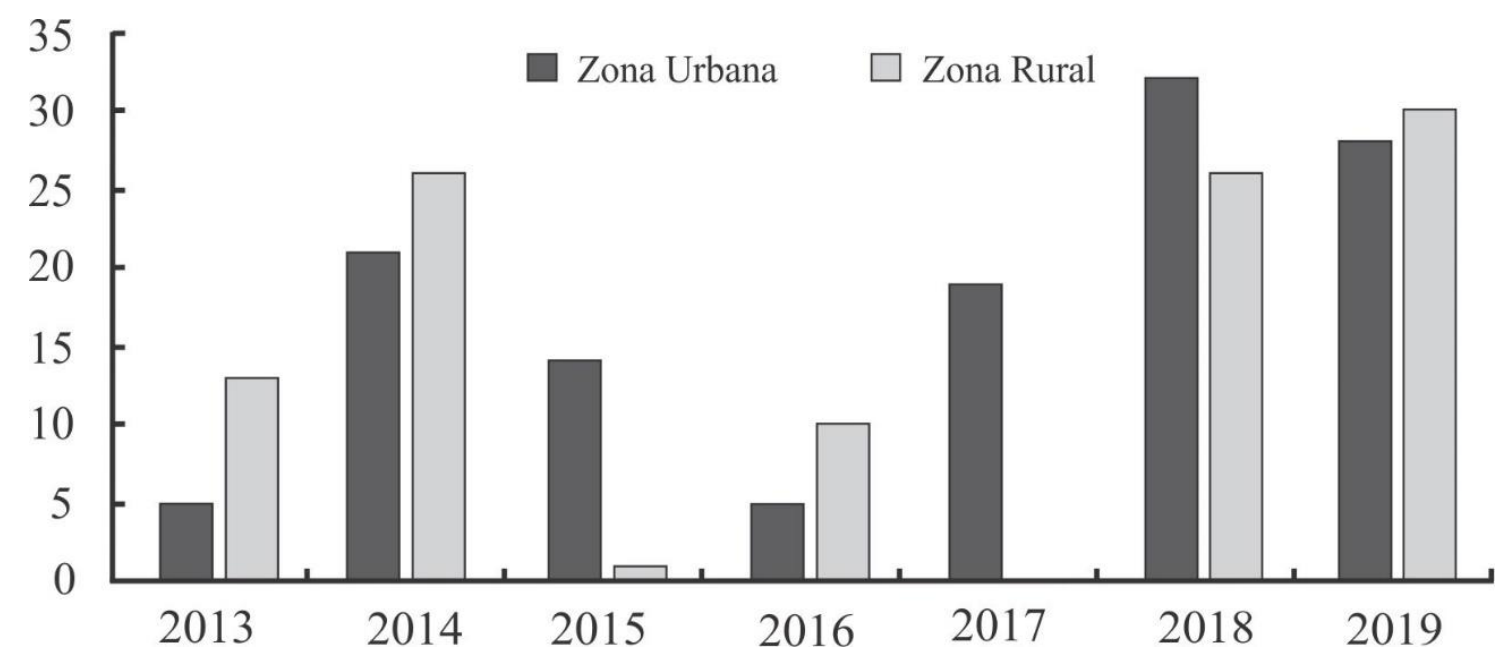

Figura 4. Índice de Prevalência da leishmaniose canina no município de Pedro II, região norte do estado do Piauí, registrados no período de 2013 a 2019.

Tabela 3. Casos de Leishmaniose Tegumentar Americana (LTA) e Leishmaniose Visceral (LV) no município de Pedro II, região norte do estado do Piauí, registrados entre os anos de 2013 a 2018.

\begin{tabular}{cccc}
\hline Ano & LTA & LV & Total \\
\hline 2013 & 01 & 03 & 04 \\
2014 & 02 & 0 & 02 \\
2015 & 10 & 02 & 12 \\
2016 & 06 & 02 & 08 \\
2017 & 11 & 02 & 13 \\
2018 & 05 & 04 & 09 \\
\hline
\end{tabular}

\section{Discussão}

Apesar de ser considerada uma doença endêmica no estado do Piauí, a LV vem tomando proporções alarmantes quando comparado ao cenário nacional, sendo registrada uma incidência (por 100 mil habitantes) de cerca de três vezes maior que a incidência registrada para todo o Brasil (Mendes et al. 2020). Nos últimos anos houve um aumento no número de trabalhos 
publicados sobre leishmaniose no Piauí (Santos et al. 2017; Lemos et al. 2018; Barroso \& Silva 2018) demostrando os elevados números de casos e evidenciando a intensa transmissão da enfermidade no estado, tanto da forma visceral quanto da tegumentar. Entre os anos de 1999 e 2009 foram confirmados no estado 2.498 casos da doença em humanos, 1.369 somente em Teresina (Drumond \& Costa 2011). Resultados publicados com dados coletados entre os anos de 2013 e 2018, metade do tempo considerado por Drumond \& Costa (2011), revelou 54\% de casos LV em relação ao estudo anterior (Mendes et al. 2020), indicando aumento do número de casos.

A grande incidência de LV no Piauí está relacionada ao fato do estado apresentar uma fauna de flebótomos representada em grande parte por Lutzomyia longipalpis, principal transmissor da LV (Soares et al. 2011), e ao grande número de cães (Drumond \& Costa 2011) vivendo próximo às áreas de ocorrência. Se o Piauí é considerado um coadjuvante na manutenção dos índices brasileiros de LV em humanos (Mendes et al. 2020), a situação é ainda mais preocupante quando se leva em conta o grande número de cães infectados com LV no estado. Somente na cidade de Teresina foram diagnosticados quase 21 mil casos da doença em cães entre os anos de 1999 e 2019 (Drumond \& Costa 2011). Contudo, apesar da importância no ciclo de transmissão em humanos, os trabalhos que abordam os aspectos epidemiológicos da doença em animais domésticos são escassos no estado (e.g., Rodrigues 2008; Santos et al. 2014; Cavalcanti et al. 2017; Mendonça et al. 2017), e abordados de forma geral ou concentrados nas grandes cidades, dificultando comparações.

Em relação à distribuição dos casos, houve diferença aparente entre as áreas urbanas e rurais do município, sendo que a zona urbana apresentou maior número de casos, mesmo com menor número de bairros em relação a quantidade de localidades rurais. Nossos resultados corroboram diversos outros artigos que evidenciam grande prevalência de cães soropositivos em áreas urbanas (Amóra et al. 2006; Barbosa et al. 2010; Pimentel et al. 2015; Silva et al. 2017). Apesar de ser originalmente endêmica de áreas rurais, estudos mostram uma urbanização da doença (Amóra et al. 2006), ocorrendo de forma endêmica e epidêmica em grandes cidades brasileiras (Werneck et al. 2002; Figueiredo et al. 2012; Silva et al. 2015; Cavalcanti et al. 2017). A alta prevalência da doença nas zonas urbanas ocorre, em grande parte, devido à explosão demográfica, ocupação desordenada e fatores ambientais que podem gerar epidemias (Freitas \& Feitosa 2014). No caso do município de Pedro II, houve grande expansão nos últimos anos, o que aproximou as zonas urbanas e rurais com a criação de novos bairros, além de ter aumento de casos caninos a partir do ano de 2017 (Tabela 1). Essa rápida expansão pode ser um dos fatores que vem contribuindo para a manutenção da doença na zona urbana.

É importante deixar claro que a leishmaniose é uma doença com perfis epidemiológicos bastante polimórficos, se caracterizando ora como doença rural, ora como doença urbana (Santos et al. 2014), o que deve ser levado em consideração pelos programas de vigilância epidemiológica. Dados publicados recentemente indicam que a LV é caracterizada como uma doença de transmissão predominantemente rural no estado de Alagoas, no qual $68 \%$ dos casos ocorreram na zona rural e apenas $32 \%$ na zona urbana (Rocha et al. 2018). Para o estado do Piauí, ainda não há caracterização geral do perfil epidemiológico da doença em relação à sua distribuição, no entanto, parece haver tendência a urbanização, como evidenciado por Rodrigues (2008) na cidade de Teresina e no município de Pedro II (presente estudo).

Em Teresina, as maiores taxas de incidência de LV humana são observadas nas regiões periurbanas, em bairros localizados na transição do ambiente rural para o urbano, no qual apresentam grande cobertura vegetal e experimentam um rápido adensamento populacional (Werneck et al. 2002; Cerbino Neto 2003; Costa 2008). Em Pedro II, os casos de cães positivos parecem seguir o mesmo padrão de ocorrência em áreas periféricas próximo à vegetação. Podese verificar na Figura 2 que a ocorrência de leishmaniose canina é maior em alguns bairros da cidade localizados próximos a áreas de mata onde os vetores são mais encontrados. $\mathrm{O}$ bairro Santa Fé, localizado próximo a áreas florestadas de vegetação nativa e com presença de pequenas nascentes, apresentou o maior número de casos da doença, cerca de $31 \%$ dos casos urbanos (Tabela 2) e possui o maior risco de contaminação da doença (Figura 2). Nestas áreas florestadas os trabalhadores rurais afirmam que há a presença de um grande número de "mosquito-palha" 
(E.B. Andrade, comunicação pessoal - novembro 2020), o que justifica o grande número de cães infectados no bairro, e a proximidade com as casas dos moradores possibilita uma transmissão da infecção humana através do ciclo silvático, não somente através do ciclo doméstico (Werneck et al. 2002). Além disso, o bairro apresenta grande número de habitações precárias e falta de saneamento básico, corroborando os dados de Cerbino Neto (2003), no qual afirma que os maiores números de casos ocorrem em áreas de ocupação desordenada e baixa infraestrutura. Apesar do bairro Centro se destacar como área de risco (Tabela 2; Figura 2), com o segundo maior número de casos, acreditamos que este número tenha sido causado pelos bairros Campestre, Cruzeiro e Saborá, localizados adjacentes ao bairro Centro e próximos às áreas de vegetação.

O município de Pedro II possui áreas em que a população humana está mais sujeita a infecção da doença devido a elevada taxa de LV canina registrada, principalmente em alguns bairros. Apesar de não haver correlação entre os casos de LV canina e LV humana no período amostrado, o cão é o principal depósito em áreas urbanas e a elevada taxa de infecção reforça a necessidade de intensificar os inquéritos sorológicos com a identificação de animais soropositivos (Guimarães et al. 2009). Em vários locais, observa-se que os casos de LV canina precedem espacial e temporalmente os casos em humanos (Rosa \& Oliveira 1997; Bevilacqua et al. 2001; Nunes et al. 2001; Guimarães et al. 2009; Troncarelli et al. 2012; Silva et al. 2015) e estima-se que para cada caso de LV humana haja em média 200 casos de LV canina (Monteiro et al. 1994). O Ministério da Saúde não recomenda que os animais positivos para a doença sejam tratados, uma vez que não há fármacos eficazes para combater a doença. Os fármacos utilizados para o tratamento em humanos não podem ser utilizados em animais pois o parasita pode adquirir resistência, tornando-se ineficaz o tratamento para humanos (Gatti et al. 2018). Com isso, o animal deve ser submetido a eutanásia e medidas de controle que reduzam a proliferação de flebotomíneos deverão ser adotadas (Gatti et al. 2018).

As variáveis epidemiológicas dos cães (raça, sexo, idade, etc.) não foram coletadas devido à falta de registro desses dados no município de Pedro II. Essas informações são importantes para traçar o perfil epidemiológico, ajudando a definir a suscetibilidade dos animais em relação à doença, já que pode haver variação em diferentes regiões. Em estudo realizado em Mossoró-RN, verificou-se que o Pitbull foi a raça mais acometida pela leishmaniose, provavelmente devido ao fato dessa raça ser levada constantemente para a realização de exames, mas não foi comprovado a predisposição para a doença (Matos et al. 2006). França-Silva et al. (2003), em estudo realizado em Minas Gerais, demonstraram que as raças mais acometidas pela LC foram Boxer e Cocker Spaniel. Por outro lado, Almeida et al. (2010) observaram que a maioria dos cães infectados eram de raça não definida/mestiços, seguida das raças Boxer, Pit Bull, Pinscher e Teckel. Apesar de alguns autores apontarem que não há predisposição epidemiológica de raças para a leishmaniose (Gontijo \& Melo 2004; Barbosa et al. 2010), observa-se que a maioria dos cães de grande porte tem uma exposição maior ao vetor (Feitosa et al. 2000; França-Silva et al. 2003; Almeida et al. 2010).

Também não há um consenso sobre a predisposição sexual dos animais quanto à infecção por leishmaniose. Alguns autores relataram não haver predisposição sexual para a doença (Feitosa et al. 2000; França-Silva et al. 2003; Matos et al. 2006), embora seja possível, em algumas localidades, associar a maior incidência aos cães machos (Caminha 2004) ou às fêmeas (Almeida et al. 2010; Silva et al. 2017). Em relação a faixa etária, apesar da probabilidade de infecção ser a mesma independentemente da idade (França-Silva et al. 2003), observa-se que a maior predominância dos casos em animais ocorre entre um a três anos de idade, devido a maior exposição ao mosquito transmissor (Matos et al. 2006). Segundo Silva et al. (2017), animais com idade acima de 12 meses e fêmeas foram mais susceptíveis à contaminação por leishmaniose devido, possivelmente, ao tempo de exposição e ao período de incubação da doença nos cães mais velhos (Brasil 2006), e ao maior risco de infecção nas fêmeas por conta das mudanças hormonais e imunológicas no período de gestação (Feliciano et al. 2012), além disso, a proximidade com galinheiros é um outro fator de risco, uma vez que acúmulo de matéria orgânica nestes locais facilita a proliferação dos flebotomíneos (Brito et al. 2016). 
Essa mudança na epidemiologia da leishmaniose canina entre as diferentes regiões do Brasil, reforça ainda mais a necessidade de montar estratégias de controle baseada nos aspectos locais de cada região. No Piauí, a transmissão da LV é intensa e os índices em humanos e cães diagnosticados são elevados, evidenciando o caráter endêmico da doença no estado.

Assim, o presente trabalho apresenta informações básicas importantes sobre a leishmaniose canina no município de Pedro II, visto que há escassez de trabalhos voltados a essa temática na região. Apesar da grande incidência de casos de leishmaniose canina no município, a doença é pouco abordada pelas autoridades de saúde e pelo poder público, levando a uma falta de conhecimento da população local sobre os procedimentos a serem seguidos em caso de suspeita de contaminação. Portanto, é necessário ações de controle por meio dos órgãos públicos e serviços de saúde do estado e do município para a realização de campanhas para o conhecimento da doença, eliminação dos flebótomos e proteção dos cães. Além disso, é fundamental a realização de trabalhos futuros para caracterizar o perfil epidemiológico da LC no município de Pedro II, permitindo um direcionamento dos esforços de controle, principalmente relacionados aos locais de maior ocorrência, susceptibilidade e exposição dos animais aos flebotomíneos, tanto dos cães domésticos quanto dos animais abandonados.

\section{Agradecimentos}

À Coordenação de Controle de Endemias de Pedro II, juntamente com o supervisor geral Rubens Galvão pela atenção e fornecimento dos dados; ao professor Hélio Ulisses por disponibilizar os dados georreferenciados para a construção dos mapas de distribuição e ocorrência e aos avaliadores pelas críticas ao manuscrito.

\section{Referências}

Aguiar R.B \& Gomes J.R.C (2004) Projeto cadastro de fontes de abastecimento por água subterrânea, estado do Piauí: diagnóstico do município de Pedro II. Serviço Geológico do Brasil. Fortaleza: CPRM. 26 p.

Almeida A.B.P.F., Mendonça A.J. \& Sousa V.R.F. (2010) Prevalência e epidemiologia da leishmaniose visceral em cães e humanos, na cidade de Cuiabá, Mato Grosso, Brasil. Ciência Rural, 40(7): 1610-1615. https://doi.org/10.1590/S0103-84782010005000102

Amóra S.S.A., Santos M.J.P., Alves N.D., Costa S.C.G., Calabrese K.S., Monteiro A.J. \& Rocha M.F.G. (2006) Fatores relacionados com a positividade de cães para leishmaniose visceral em área endêmica do Estado do Rio Grande do Norte, Brasil. Ciência Rural, 36(6): 1854-1859. https://doi.org/10.1590/S0103-84782006000600029

Barbosa D.S., Rocha A.L., Santana A.A., Souza C.S.F., Dias R.A., Costa-Júnior L.M. \& Abreu-Silva A.L. (2010) Soroprevalência e variáveis epidemiológicas associadas à leishmaniose visceral canina em área endêmica no município de São Luís, Maranhão, Brasil. Ciência Animal Brasileira, 11(3): 653-659. https://doi.org/10.5216/cab.v11i3.5933

Barroso A.M.C. \& Silva A.K.M. (2018) Perfil clínico e epidemiológico da leishmaniose no estado do Piauí no período de 2007 a 2017. Monografia (Graduação em Biomedicina). Centro Universitário UNINOVAFAPI, Teresina, Piauí.

Batista F.M.A., Machado F.F.O.A., Silva J.M.O., Mittmann J., Barja P.R. \& Simioni A.R. (2014) Leishmaniose: perfil epidemiológico dos casos notificados no estado do Piauí entre 2007 e 2011. Revista Univap, 20(35): 44-55. http://dx.doi.org/10.18066/revunivap.v20i35.180

Bavia M.E., Carneiro D.D.M.T., Gurgel H.C., Filho C.M. \& Barbosa M.G.R. (2005) Remote sensing and geographic information systems and risk of American visceral leishmaniasis in Bahia, Brazil. Parasitology, 47(3): 165-169.

Bevilacqua P.D., Paixão H.H., Modena C.M. \& Castro M.C.P.S. (2001) Urbanização da leishmaniose visceral em Belo Horizonte. Arquivo Brasileiro de Medicina Veterinária e Zootecnia, 53(1): 1-8. https://doi.org/10.1590/S0102-09352001000100001

Bonates A. (2003) Leishmaniose Visceral (Calazar). Veterinary News, 61: 4-5. 
Brasil (2006) Ministério da Saúde. Manual de vigilância e controle da Leishmaniose Visceral. Secretaria de Vigilância em Saúde. Departamento de Vigilância Epidemiológica. Série A. Normas e Manuais Técnicos. Brasília: Editora do Ministério da Saúde. 120 p.

Brito F.G., Langoni H., Silva R.C., Rotondano T.E.F., Melo M.A. \& Paz G.S. (2016) Canine visceral leishmaniasis in the Northeast Region of Brazil. Journal of Venomous Animals and Toxins including Tropical Diseases, 22(15): 1-4. https://doi.org/10.1186/s40409-016-0069-4

Caldas A.J.M., Lisbôa L.L.C., Silva P.F., Coutinha N.P.S. \& Silva T.C. (2013) Perfil das crianças com leishmaniose visceral que evoluíram para óbito, falha terapêutica e recidiva em hospital de São Luís, Maranhão. Revista de Pesquisa em Saúde, 14(2): 91-95.

Caminha A.E.Q. (2004) Aspectos clínicos da leishmaniose visceral canina na cidade de Fortaleza, Ceará. Monografia (Graduação em Medicina Veterinária). Universidade Federal Rural do Semi-Árido, Mossoró, Rio Grande do Norte.

Cavalcanti O.L., Miranda V.L., Lappa F.P.F., Barbosa D.B.S., Fortier D.C., Nascimento L.M.F., Barbosa D.R.S. \& Soares M.R.A. (2017) Aspectos da incidência de leishmaniose visceral humana e canina no município de Floriano/PI, Brasil. Revista Espacios, 38(8): 20-26.

Cerbino Neto J. (2003) Fatores Associados à Incidência de Leishmaniose Visceral em Teresina-PI na Década de 90. Dissertação (Programa de Pós-Graduação em Medicina). Universidade Federal do Rio de Janeiro, Faculdade de Medicina, Rio de Janeiro.

Costa C.H.N. (2008) Characterization and speculations on the urbanization of visceral leishmaniasis in Brazil. Cadernos de Saúde Pública, 24(12): 2959-2963. http://dx.doi.org/10.1590/S0102-311X2008001200027

Drumond K.O. \& Costa F.A.L. (2011) Forty years of visceral leishmaniasis in the State of Piaui: a review. Revista do Instituto de Medicina Tropical de São Paulo, 53(1): 3-11. http://dx.doi.org/10.1590/S0036-46652011000100002

Feitosa M.M., Ikeda F.A., Luvizotto M.C.R. \& Perri S.H.V. (2000) Aspectos clínicos de cães com leishmaniose no município de Araçatuba, São Paulo (Brasil). Clínica Veterinária, 28: 36-44.

Feliciano M.A.R., Aquino A.A., Coutinho L.N. \& Vicente W.R.R. (2012) Imunologia na gestação de cadelas: revisão de literatura. Revista Brasileira de Reprodução Animal, 36: 158-162.

Figueiredo F.B., Lima Júnior F.E.F., Tomio J.E., Indá F.M.C., Corrêa G.L.B. \& Madeira M.F. (2012) Leishmaniose visceral canina: dois casos autóctones no município de Florianópolis, estado de Santa Catarina. Acta Scientiae Veterinariae, 40(1): 1026.

França-Silva J.C., Costa R.T., Siqueira A.M., Machado-Coelho G.L.L., Costa C.A., Mayrink W., Vieira E.P., Costa J.S., Genaro O. \& Nascimento E. (2003) Epidemiology of canine visceral leishmaniasis in the endemic area of Montes Claros Municipality, Minas Gerais state, Brazil. Veterinary Parasitology, 111: 161-173. http://dx.doi.org/10.1016/s0304-4017(02)00351-5

Freitas J.C.C. (2011) Subsídios para o estudo da leishmaniose visceral canina na cidade de Fortaleza, Ceará. Tese (Programa de Pós-Graduação em Ciências Veterinárias). Universidade Estadual do Ceará, Faculdade de Veterinária, Fortaleza, Ceará.

Freitas L.C.S. \& Feitosa A.C. (2014) Espaço e Saúde: condições socioambientais favoráveis à leishmaniose visceral (LV) na bacia do rio Anajá em Paço do Lumiar - MA. Revista Brasileira de Geografia Médica e da Saúde, 10(18): 33-45.

Freitas S.O., Gomes J.M.A. \& Aquino C.M.S. (2016) Análise dos impactos ambientais da extração de opala no munícipio de Pedro II, Piauí. Geociências, 35(3): 443-456.

Gatti R.R., Matsumoto A.M.B. \& Simom Y.G. (2018) Guia de orientação para vigilância de leishmaniose visceral canina (LVC). Florianópolis: Secretaria de Estado da Saúde de Santa Catarina. 40 p.

Gontijo C.M.F. \& Melo M.N. (2004) Leishmaniose visceral no Brasil: quadro atual, desafios e perspectivas. Revista Brasileira de Epidemiologia, 7(3): 338-349. https://doi.org/10.1590/S1415-790X2004000300011

Guimarães M.A., Rocha C.M.B.M., Oliveira T.M.F.S., Rosado I.R., Morais L.G. \& Santos R.R.D. (2009) Fatores associados à soropositividade para Babesia, Toxoplasma, Neospora e Leishmania em cães atendidos em nove clínicas veterinárias do município de Lavras, MG. Revista Brasileira de Parasitologia Veterinária, 18: 49-53. 
https://doi.org/10.4322/rbpv.018e1009

Hammer O., Harper D.A.T. \& Ryan P.D. (2001) PAST: Paleontological Statistic software package for education and data analysis. Paleontologia Eletronica, 4(1): 1-9.

IBGE (2019) Instituto Brasileiro de Geografia e Estatística. Biomas e sistema costeiro-marinho do Brasil: compatível com a escala 1:250 000. Coordenação de Recursos Naturais e Estudos Ambientais. Rio de Janeiro: IBGE. Série Relatórios Metodológicos, 45: 168.

IBGE (2021) Instituto Brasileiro de Geografia e Estatística. Cidade: Pedro II. Instituto Brasileiro de Geografia e Estatística. Disponível em: http://cidades.ibge.gov.br (Acessado em 11/01/2021).

ISA (2017) Instituto Socioambiental. Unidades de Conservação do Brasil: APA da Serra da Ibiapaba. Disponível em: http://uc.socioambiental.org (Acessado em 10/10/2020).

Lemos M.H.S., Silva W.C., Gomes F.C.S., Lages L.P., Costa J.O., Júnior J.D.P.A., Teixeira D.R.A., Santos F.A.S., Pinheiro D.H.A. \& Queiroz B.F.S. (2018) Epidemiologia das Leishmanioses no estado do Piauí. Brazilian Journal of Surgery and Clinical Research, 25(2): 53-57.

Lima M.L.C., Ximenes R.A.A, Souza E.R., Luna C.F. \& Albuquerque M.F.P.M. (2005) Análise espacial dos determinantes socioeconômicos dos homicídios no Estado de Pernambuco. Revista de Saúde Pública, 39(2): 176-82. http://dx.doi.org/10.1590/S0034-89102005000200006

Marcondes M. \& Rossi C.N. (2013) Leishmaniose visceral no Brasil. Brazilian Journal of Veterinary Research and Animal Science, 50(5): 341-352.

Matos M.M., Filgueira K.D., Amora S.S.A., Suassuna A.C.D., Ahid S.M.M. \& Alves N.D. (2006) Ocorrência da leishmaniose visceral em cães em Mossoró, Rio Grande do Norte. Ciência Animal, 16(1): 51-54.

Mendes J.R., Lopes A.S., Sousa M.S.C., Silva M.J.M., Sousa P.B., Chagas N.S., Marcelo Ventura C.S., Silva D.F.M., Mallet J.R.S., Vilela M.L. \& Silva J. (2020) O Piauí como coadjuvante da leishmaniose visceral brasileira. Brazilian Journal of Development, 6(3): 11210-11219. http://dx.doi.org/10.34117/bjdv6n3-114

Mendonça I.L., Batista J.F., Ribeiro I.M.M., Rocha F.S.B., Silva S.O. \& Melo M.N. (2017) Leishmania infantum in domestic cats from the municipality of Teresina, state of Piauí, Brazil. Parasitology Open, 3: 1-8.

Monteiro P., Lacerda M.M. \& Arias J.R. (1994) Controle da Leishmaniose no Brasil. Revista da Sociedade Brasileira de Medicina Tropical, 27(4): 67-72.

Nunes V.L.B., Galati E.A.B., Nunes D.B., Zinezzi R.O., Savani E.S.M.M., Ishikawa E., Camargo M.C.G.O., D'Áuria S.R.N., Cristaldo G. \& Rocha H.C. (2001) Ocorrência de leishmaniose visceral canina em assentamento agrícola no Estado de Mato Grosso do Sul, Brasil. Revista da Sociedade Brasileira de Medicina Tropical, 34: 301-302.

http://dx.doi.org/10.1590/S0037-86822001000300014

Pimentel D.S., Ramos R.A.N., Santana M.A., Maia C.S., Carvalho G.A., Silva H.P. \& Alves L.C. (2015) Prevalence of zoonotic visceral leishmaniasis in dogs in an endemic area of Brazil. Revista da Sociedade Brasileira de Medicina Tropical, 48(4): 491-493. http://dx.doi.org/10.1590/0037-8682-0224-2014

QGis Development Team (2020) Quantum GIS Geographic Information System. Open Source Geospatial Foundation Project. Disponível em: http://qgis.osgeo.org (Acessado em: 10/10/2020).

Rocha M.A.N., Matos-Rocha T.J., Ribeiro C.M.B. \& Abreu S.R.O. (2018) Epidemiological aspects of human and canine visceral leishmaniasis in State of Alagoas, Northeast, Brazil. Brazilian Journal of Biology, 78(4): 609-614. http://dx.doi.org/10.1590/1519-6984.166622

Rodrigues A.C.E. (2008) Características epidemiológicas e distribuição espacial da enzootia canina de Leishmaniose Visceral na cidade de Teresina - Piauí, no período de 2003 a 2006. Dissertação (Programa de Pós-Graduação em Saúde Pública). Fundação Oswaldo Cruz, Escola Nacional de Saúde Pública Sergio Arouca, Rio de Janeiro.

Rosa I.C.A.S. \& Oliveira I.C.S. (1997) Leishmaniose visceral: breve revisão sobre uma zoonose reemergente. Clínica Veterinária, 11:24-28. 
Santos J.P., Dias-e-Silva T.P., Lima D.W.G. \& Mendonça I.L. (2014) Leishmaniose visceral no município de Bom Jesus, Piauí, Brasil. Acta Veterinária Brasílica, 8(4): 236-241. https://doi.org/10.21708/avb.2014.8.4.4302

Santos G.M., Barreto M.T.S., Monteiro M.J.S.D., Silva R.V.S., de Jesus R.L.R. \& Silva H.J.N. (2017) Aspectos epidemiológicos e clínicos da leishmaniose visceral no estado do Piauí, Brasil. Ciência \& Desenvolvimento-Revista Eletrônica da FAINOR, 10(2): 142-153. http://dx.doi.org/10.11602/1984-4271.2017.10.2.12

Silva D.A., Madeira M.F. \& Figueiredo F.B. (2015) Geographical Expansion of canine visceral leishmaniasis in Rio de Janeiro state, Brazil. Revista do Instituto de Medicina Tropical, 57(5): 435-438. https://doi.org/10.1590/S0036-46652015000500012

Silva A.V.M.D., Paula A.A.D., Cabrera M.A.A. \& Carreira J.C.A. (2005) Leishmaniose em cães domésticos: aspectos epidemiológicos. Cadernos de Saúde Pública, 21: 324-328. https://doi.org/10.1590/S0102-311X2005000100036

Silva J.D., Melo D.H.M., Costa J.A.G., Costa D.F., Silva R.B.S., Melo M.A., Azevedo S.S. \& Alves C.J. (2017) Leishmaniose visceral em cães de assentamentos rurais. Pesquisa Veterinária Brasileira, 37(11): 1292-1298. https://doi.org/10.1590/s0100-736x2017001100016

Siqueira F.R.D. (2012) Leishmaniose visceral canina. Monografia (Especialização em Clínica Médica e Cirúrgica de Pequenos Animais). UNIGRAN - Centro Universitário da Grande Dourados, Mato Grosso do Sul.

Soares M.R.A., Mendonca I.L., Bonfim J.M., Rodrigues J.A., Werneck G.L. \& Costa C.H.N. (2011) Canine visceral leishmaniasis in Teresina, Brazil: Relationship between clinical features and infectivity for sand flies. Acta Tropical, 117: 6-9. https://doi.org/10.1016/j.actatropica.2010.08.015

Tilley L.P. \& Smith Jr. F.W.K. (2008) Consulta veterinária em cinco minutos. Espécies canina e felina. $3^{\circ}$ edição. São Paulo: Manole. 1550 p.

Troncarelli M.Z., Carneiro D.M.V.F. \& Langoni H. (2012) Visceral Leishmaniosis: an old disease with continuous impact on Public Health (p. 263-282). In: Lorenzo-Morales J. (Ed.). Zoonosis. InTech. https://doi.org/10.5772/2125

Werneck G.L., Costa C.H.N., Walker A.M., David J.R., Wand M. \& Maguire J.H. (2002) The urban spread of visceral leishmaniasis: Clues from spatial analysis. Epidemiology, 13(3): 364-367. https://doi.org/10.1097/00001648-200205000-00020

Werneck G.L., De Jesus T.F.P.C., Farias G.C., Silva F.O., Chaves F.C., Golvêa M.V., Henrique C.N.C. \& Amorim F.C.A. (2008) Avaliação da efetividade das estratégias de controle da leishmaniose visceral na cidade de Teresina, Estado do Piauí, Brasil: resultados do inquérito inicial - 2004. Epidemiologia e Serviços de Saúde, 17(2): 87-96.

Zar J.H. (2010) Biostatistical analysis. $5^{\circ}$ edição. New York: Prentice - Hall/Pearson. 944 p. 